\title{
Thermal Conductivity of Ionic Systems from Equilibrium Molecular Dynamics
}

\author{
Mathieu Salanne ${ }^{1}$, Dario Marrocchelli ${ }^{2,3}$, Céline Merlet $^{1,4}$, \\ Norikazu Ohtori ${ }^{5}$ and Paul A. Madden ${ }^{4}$ \\ ${ }^{1}$ UPMC Univ-Paris06 and CNRS, UMR 7195, PECSA, F-75005, Paris, \\ France \\ ${ }^{2}$ School of Chemistry, University of Edinburgh, Edinburgh EH9 3JJ, UK \\ ${ }^{3}$ Department of Nuclear Science and Engineering, Massachusetts Institute of \\ Technology, 77 Massachusetts Ave., Cambridge, MA 02139, USA \\ ${ }^{4}$ Department of Materials, University of Oxford, Parks Road, Oxford OX1 \\ $3 \mathrm{PH}, \mathrm{UK}$ \\ ${ }^{5}$ Graduate School of Science and Technology, Niigata University, Niigata \\ 950-2181, Japan \\ E-mail: mathieu.salanne@upmc.fr
}

\begin{abstract}
Thermal conductivities of ionic compounds ( $\mathrm{NaCl}, \mathrm{MgO}, \mathrm{Mg}_{2} \mathrm{SiO}_{4}$ ) are calculated from equilibrium molecular dynamics simulations using the GreenKubo method. Transferable interaction potentials including many-body polarization effects are employed. Various physical conditions (solid and liquid states, high temperatures, high pressures) relevant to the study of the heat transport in the Earth's mantle are investigated, for which experimental measures are very challenging. By introducing a frequency-dependent thermal conductivity, we show that important coupled thermoelectric effects occur in the energy conduction mechanism in the case of liquid systems.
\end{abstract}


Knowledge of the thermal properties of ionic materials under extreme conditions has become a focus of attention in several fields of study. The thermal conductivity, which measures a material's capability to transport heat, is of particular interest; it is also the most difficult to measure experimentally at high pressures [1] and high temperatures [2]. Its computation is also very challenging [3,4]. For these reasons, very few data are available. Lack of knowledge of the thermal conductivity of minerals at the temperatures and pressures relevant to the Earth's mantle is a significant barrier to quantitative understanding of the heat flux from the earth's core to the surface $[5,6]$. Several new energy-related technologies also require information on the thermal conductivity of ionic melts at high temperatures. In the Generation IV nuclear reactor concepts, the Advanced High-Temperature Reactor (AHTR) [7] and the Molten Salt Fast Reactor (MSR) [8], a molten fluoride acts as coolant. Molten salts are also proposed for heatexchangers in solar thermal and fusion power plants. In these technologies values for the thermal conductivity are required for engineering calculations of the proposed plant performance.

In this work, we use some recently introduced expressions for the energy flux [3] to calculate thermal conductivities of ionic compounds from classical molecular dynamics simulations. The method is particularly appropriate to high temperatures where quantum effects are negligible and where an approach based upon a calculation of the vibrational modes cannot be applied because of dominant anharmonic effects or melting. Here we focus first on $\mathrm{NaCl}$, for which we study both the pressure effect on the solid state thermal conductivity and the difference of behaviour between the solid and liquid states. We then determine the thermal conductivities of two oxide systems in the liquid state, namely $\mathrm{MgO}$ and $\mathrm{Mg}_{2} \mathrm{SiO}_{4}$. Our approach involves two key aspects: First, we employ interaction potentials that include many-body effects and were parameterized from first-principles calculations $[3,9]$. This allows us to save a lot of computational effort compared to purely first-principles based approaches [10, 11]. Second, we determine the thermal conductivity from equilibrium molecular dynamics simulations, using the Green-Kubo method.

Ionic compounds are thermodynamic systems in which irreversible processes of thermal conduction, diffusion and electric conduction arise due to the existence of temperature, chemical potential and electric potential gradients, and important coupled thermoelectric effects occur. The entropy production for a mixture containing $N$ ionic species is given by [12]:

$$
\sigma=-\frac{1}{T^{2}} \mathbf{J}_{Q} \cdot \boldsymbol{\nabla} T-\frac{1}{T} \sum_{i=1}^{N} \mathbf{J}_{i} \cdot \boldsymbol{\nabla}_{T} \mu_{i}-\frac{1}{T} \mathbf{J}_{Z} \cdot \boldsymbol{\nabla} \phi
$$

where $\mathbf{J}_{a}$ designates the nonconvective fluxes of heat $(a=Q)$, mass $(i)$ and charge $(Z)$; the subscript $T$ means that the chemical potential gradient has to be taken at constant temperature. In computer simulations, the calculation of the microscopic energy flux $\mathbf{j}_{E}$ is more convenient than its heat counterpart. We therefore introduce the macroscopic 
energy flux defined by

$$
\mathbf{J}_{E}=\mathbf{J}_{Q}+\sum_{i=1}^{N} \tilde{h}_{i} \mathbf{J}_{i}
$$

where $\tilde{h}_{i}$ is the partial specific enthalpy of component $i$, in the presence of an electric field $\phi, \tilde{h}_{i}=h_{i}+z_{i} \phi$ (this quantity parallels the electrochemical potential $\tilde{\mu}_{i}=\mu_{i}+z_{i} \phi$ ). In systems containing one anionic species only (which will be labeled $N$ ), following the approach proposed in reference [13] it is also useful to introduce the fluxes

$$
\mathbf{J}_{Z_{i}}=\left(z_{i}-z_{N}\right) \mathbf{J}_{i}, i \in[1, N-1] .
$$

In the linear response regime, these fluxes can be written as a set of linear equations:

$$
\begin{aligned}
& \mathbf{J}_{E}=-\frac{L_{E E}}{T^{2}} \nabla T-\sum_{j=1}^{N-1} \frac{L_{E Z_{j}}}{T} \nabla\left(\frac{\mu_{j}^{\prime}}{T}\right) \\
& \mathbf{J}_{Z_{i}}=-\frac{L_{Z_{i} E}}{T^{2}} \nabla T-\sum_{j=1}^{N-1} \frac{L_{Z_{i} Z_{j}}}{T} \nabla\left(\frac{\mu_{j}^{\prime}}{T}\right)
\end{aligned}
$$

where $\mu_{i}^{\prime}=\left(\tilde{\mu}_{i}-\tilde{\mu}_{N}\right) /\left(z_{i}-z_{N}\right)$ and $L_{a b}$ are the phenomenological coefficients. For the purpose of present work, it is useful to introduce the frequency-dependent quantities:

$$
L_{a b}^{\omega}=\frac{1}{3 V k_{B}} \int_{0}^{\infty}\left\langle\mathbf{j}_{a}(t) \cdot \mathbf{j}_{b}(0)\right\rangle \exp (\imath \omega t) \mathrm{d} t .
$$

The frequency-dependent thermal conductivity is then given, for a binary ionic mixture [14, 13], by:

$$
\lambda(\omega)=T^{-2}\left(L_{E E}^{\omega}-\frac{\left(L_{E Z_{1}}^{\omega}\right)^{2}}{L_{Z_{1} Z_{1}}^{\omega}}\right)
$$

In the most recent work on inorganic molten salts, the interactions between the ions are described by polarizable interaction potentials which are parameterized on the basis of first-principles electronic structure calculations [15]. Such potentials have been shown to reproduce bulk structural, thermodynamic and transport properties extremely well $[16,3]$; they are predictive in the sense that no empirical information is used in their construction and highly transferable from pure materials to mixtures. The potentials are constructed by a generalized "force-matching" method. A suitable condensedphase ionic configuration is taken from a molecular dynamics simulation using some approximate force-field for the material of interest. Typically a hundred ions would be used in periodic boundary conditions. The configuration is then input to a planewave DFT electronic structure program and an energy minimization carried out to find the ground-state electronic structure. From the results of this calculation the force and dipole moment on each ion is obtained, the latter by making use of the transformation of the Kohn-Sham orbitals to a Maximally Localized Wannier Function (MLWF) set. The parameters in the polarizable potential are then optimized by matching the dipoles and forces from the potential on the same ionic configuration to the ab initio values [15]. If necessary the process may be iterated, by using the fitted potential to generate a new 

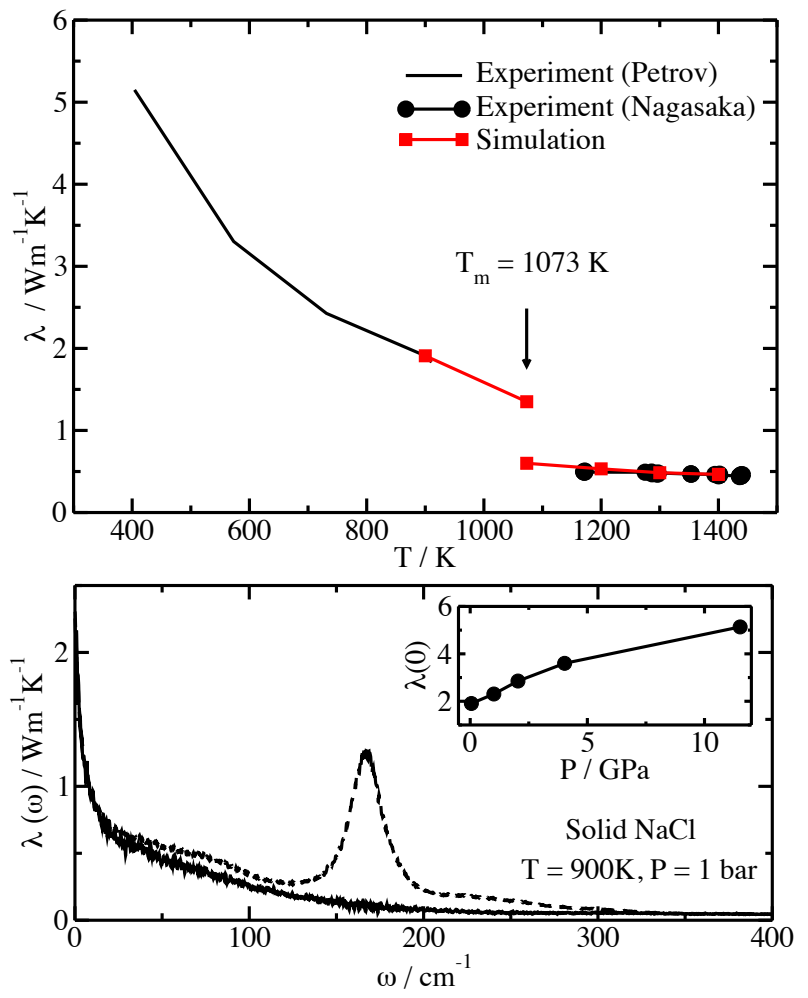

Figure 1. (color online) Upper panel: Thermal conductivity of solid and liquid $\mathrm{NaCl}$ $\left(T_{m}=1073 \mathrm{~K}\right)$. Experimental values are taken from references [2] and [20]. Lower panel: Frequency-dependent thermal conductivity of solid $\mathrm{NaCl}$ at $T=900 \mathrm{~K}$ and $P=1 \mathrm{GPa}$ (solid line), $L_{E E}^{\omega} / T^{2}$ for the same system (dashed line). Insert: Thermal conductivity $\lambda(\omega=0)$ (same units) as a function of pressure in solid $\mathrm{NaCl}$ at $T=900 \mathrm{~K}$.

ionic configuration to input to the ab initio calculation. The resulting potentials may be used in much larger scale MD simulations to obtain the physical properties of interest $[17,18]$. In the case of simple systems, the "force-matching" may even be avoided by computing the various interaction terms separately [19].

We first focus on $\mathrm{NaCl}$, for which experimental data are available in both the liquid and solid states. We made use of the interaction potentials and methods described in reference [3], and the simulation details are given as a supplementary information. Our results are summarized on figure 1 . They agree very well with experimental data at zero pressure $[2,20]$. Standard errors of $0.07 \mathrm{~W} \cdot \mathrm{m}^{-1} \cdot \mathrm{K}^{-1}$ (solid at $0 \mathrm{GPa}$ ) and $0.02 \mathrm{~W} . \mathrm{m}^{-1} . \mathrm{K}^{-1}$ (liquid) were determined following reference [21]. Upon pressurization of the solid at $900 \mathrm{~K}$, we observe an increase of the thermal conductivity, which reaches a value of $5.13 \pm 0.19 \mathrm{~W} . \mathrm{m}^{-1} \cdot \mathrm{K}^{-1}$ at $P=11.5 \mathrm{GPa}$. This increase is more pronounced compared to the values proposed by MacPherson et al. [22]. Unfortunately, this is the only experimental data available at $900 \mathrm{~K}$ at high pressure; by comparison with other authors' data at room temperature it appears that a serious systematic error was not taken into account in this work [23]. Hofmeister has shown that the measured thermal conductivity varies with the number of physical contacts between the sample and heaters 
and/or thermocouples [1]. The discrepancy between our value and McPherson's at high pressure is of the same size as that seen between his value and that reported by others at ambient pressure and attributed to this source of error [23].

In the recent work focused on the first-principles calculation of thermal conductivities in ionic crystals $(\mathrm{MgO})$, two different approaches have been proposed. The first consists of determining the phonon lifetimes and frequencies [10]. It involves relatively few computations and therefore seems more adapted to such materials than our equilibrium molecular dynamics method, which requires typical simulation times of a few tenth of ns in the case of simple solid systems. It will nevertheless suffer from drawbacks in some important applications: For complex solids, the evaluation of anharmonic phonon frequencies becomes much more difficult, and in the case of liquid systems the method cannot be applied. The second method, proposed by Stackhouse et al., builds upon past work on non-equilibrium molecular dynamics methods. A timedependent energy flux $j_{E}^{x}(t)$ is imposed across the simulation cell, and the thermal conductivity is extracted using Fourier's law:

$$
\lambda^{\prime}(0)=\frac{\left\langle j_{E}^{x}\right\rangle}{\langle\partial T / \partial x\rangle}
$$

where we have used the notation $\lambda^{\prime}$ because it neglects the coupled thermoelectric effects corresponding to the second term of equation 7 . This approach therefore is valid for ionic insulator systems only. On the bottom panel of figure 1, we show the comparison between $\lambda(\omega)$ and $\mathrm{E}_{E E}^{\omega} / T^{2}$ for $\mathrm{T}=900 \mathrm{~K}$ and $P=1 \mathrm{GPa}$. It can be seen that the two curves match very well apart from an intense peak centered at $\omega=167 \mathrm{~cm}^{-1}$. The latter corresponds to fluctuations of the electric current at transverse optic frequency. At lower frequencies, the two set of data merge, therefore validating the approach of Stackhouse et al. for crystalline systems like $\mathrm{NaCl}$ and $\mathrm{MgO}$. In the case of ionic conductor crystals like $\mathrm{CaF}_{2}$ and $\mathrm{UO}_{2}$, it was shown by Lindan and Gillan that the two terms of equation 7 strongly cancel each other, preventing the use of non-equilibrium molecular dynamics for such systems [24].

In the case of ionic liquids, it is very likely that thermoelectric effects will be very important. Surprisingly, it was shown by Galamba et al., from a direct comparison between equilibrium and non-equilibrium classical molecular dynamics simulations, that the two methods provided results in good agreement one with each other for molten $\mathrm{NaCl}$ and $\mathrm{KCl}[13]$. This result is largely due to the fact that in these systems the second-term of equation 7 remains small, which would not be the case in molten $\mathrm{LiCl}$ [3]. Here we will consider liquids that are relevant for the study of Earth mantle, namely $\mathrm{MgO}$ and $\mathrm{Mg}_{2} \mathrm{SiO}_{4}$ (forsterite). We stress that our calculation do not include the contribution from radiative effects or the electronic mechanism, which are expected to be important at the temperatures of interest ( $2800 \mathrm{~K}$ and $3500 \mathrm{~K}$ respectively); these terms can be calculated separately [5]. The interaction potentials needed to describe the oxides are more sophisticated than the dipole-polarizable potentials which suffice for halides. The many-body polarization part includes dipolar and quadrupolar degrees of freedom, while the short-range repulsion takes into account an asymmetric shape deformations of the 


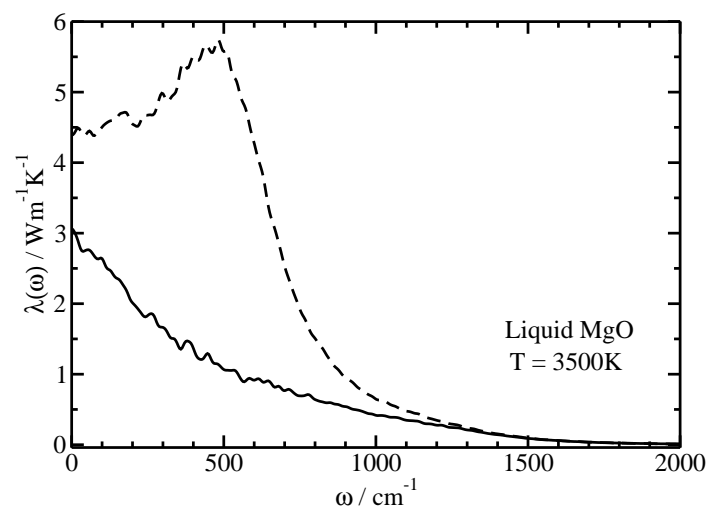

Figure 2. Frequency dependent thermal conductivity (solid line) and $L_{E E}^{\omega} / T^{2}$ (dashed line) for liquid $\mathrm{MgO}$ at $T=3500 \mathrm{~K}$.

oxide ions. Here again, the parameters were obtained from first-principles calculations, and no experimental data were used in the parameterization. The model reproduces accurately lattice parameters and thermoelastic properties of minerals in a wide range of compositions [9], and has been shown to be transferable in a wide range of pressure and temperature [25], which means that we can be confident in their ability to predict quantities that have never been measured, like the thermal conductivities in the liquid state.

The frequency-dependent thermal conductivity obtained for liquid $\mathrm{MgO}$ at $T=3500 \mathrm{~K}$ is displayed on figure 2 . We calculate a thermal conductivity of $3.06 \pm 0.2 \mathrm{~W} \cdot \mathrm{m}^{-1} \cdot \mathrm{K}^{-1}$. In this case, an evaluation based on the energy flux based term only would provide a value of around $4.39 \mathrm{~W} \cdot \mathrm{m}^{-1} \cdot \mathrm{K}^{-1}$, i.e. an error of $43 \%$. The origin of this difference is clearly seen on figure 2 : The $L_{E E}^{\omega} / T^{2}$ term presents an intense peak centered at a frequency of $480 \mathrm{~cm}^{-1}$, which is broad enough to still be appreciable at the smallest frequencies which are relevant for the thermal conductivity itself. The peak is due to fluctuations of the electric current and is therefore projected out by the second term of equation 7 in $\lambda(\omega)$. This means that, unlike molten $\mathrm{NaCl}$ or $\mathrm{KCl}$, it is very unlikely that a non-equilibrium molecular dynamics will provide a reliable result in this case.

Even more interesting is the example of liquid $\mathrm{Mg}_{2} \mathrm{SiO}_{4}$. In this case, the thermal conductivity is given by

$$
\lambda(\omega)=T^{-2}\left(L_{E E}^{\omega}-\frac{A}{B}\right)
$$

where

$$
\begin{aligned}
A= & \left(L_{E Z_{1}}^{\omega}\right)^{2} L_{Z_{2} Z_{2}}^{\omega}+\left(L_{E Z_{2}}^{\omega}\right)^{2} L_{Z_{1} Z_{1}}^{\omega} \\
& -2 L_{E Z_{1}}^{\omega} L_{E Z_{2}}^{\omega} L_{Z_{1} Z_{2}}^{\omega} \\
B= & L_{Z_{1} Z_{1}}^{\omega} L_{Z_{2} Z_{2}}^{\omega}-\left(L_{Z_{1} Z_{2}}^{\omega}\right)^{2}
\end{aligned}
$$




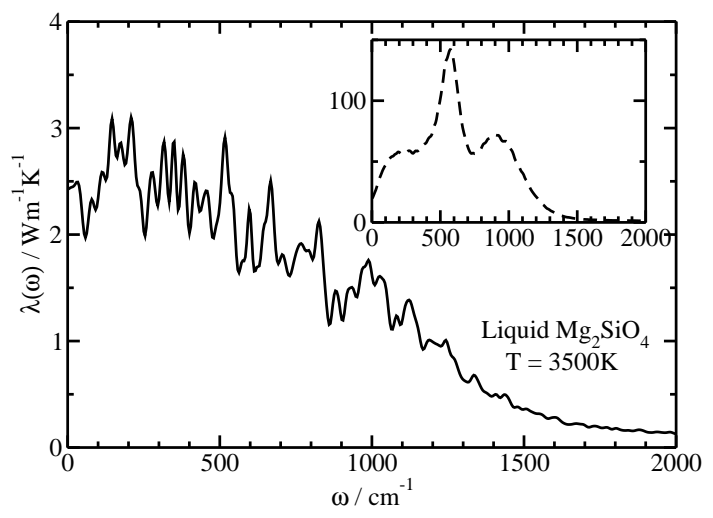

Figure 3. Frequency dependent thermal conductivity for liquid forsterite $\left(\mathrm{Mg}_{2} \mathrm{SiO}_{4}\right)$ at $T=2800 \mathrm{~K}$. Inset: $L_{E E}^{\omega} / T^{2}$ (same units).

The frequency-dependent thermal conductivity at $T=2800 \mathrm{~K}$ is given in figure 3 . We calculate a thermal conductivity of $2.4 \pm 0.4 \mathrm{~W} \cdot \mathrm{m}^{-1} \cdot \mathrm{K}^{-1}$. The important error bar in this case is due to the need to determine six different auto-correlation functions, and to the very strong cancellation between the two terms of equation 9: The neglect of thermoelectric effects would lead to a value of around $20 \mathrm{~W} \cdot \mathrm{m}^{-1} \cdot \mathrm{K}^{-1}$, which is physically unrealistic for such a liquid. As shown in the inset of figure 3, this is again due to some fluctuations of the electric current; which are enhanced by almost two order of magnitudes compared to the case of liquid $\mathrm{MgO}$ because they include the internal vibrations of the $\mathrm{SiO}_{4}$ tetrahedral entities present in the melt [26].

In conclusion, we have shown in this paper that equilibrium molecular dynamics simulation remains the method of choice for determining thermal conductivities in ionic systems at high temperatures. This is at the price of substantial work on the parameterization of reliable and transferable interaction potentials. This approach also presents the advantage of providing a full set of thermodynamic and transport properties (viscosity, heat capacity, diffusion coefficients...) within one single simulation. In the case of solid systems, large simulation times are needed, but this drawback is largely compensated by the much lower computational price compared to fully first-principles methods.

This research was partially supported by CNRS through PCR-ANSF and PACEN programmes. The work has also been performed under the HPC-EUROPA2 project (project number: 228398) with the support of the European Commission -Capacities Area- Research Infrastructures. M.S. is grateful to Niigata University for a travel grant which enabled this collaboration.

[1] A. M. Hofmeister. Pressure dependence of thermal transport properties. Proc. Natl. Acad. Sci. U.S.A., 104(22):9192-9197, 2007.

[2] Y. Nagasaka, N. Nakazawa, and A. Nagashima. Experimental determination of the thermal diffusivity of molten alkali halides by the forced rayleigh scattering method. 1. molten LiCl, 
$\mathrm{NaCl}, \mathrm{KCl}, \mathrm{RbCl}$ and $\mathrm{CsCl}$. Int. J. Thermophys., 13(4):555-574, 1992.

[3] N. Ohtori, M. Salanne, and P.A. Madden. Calculations of the thermal conductivities of ionic materials by simulation with polarizable interaction potentials. J. Chem. Phys., 130(10):104507, 2009.

[4] Y. Long, J. Chen, Y. G. Liu, F. D. Nie, and J. S. Sun. A direct method to calculate thermal conductivity and its application in solid hmx. J. Phys.: Condens. Matter, 22(18):185404, 2010.

[5] A. M. Hofmeister. Mantle values of thermal conductivity and the geotherm from phonon lifetimes. Science, 283:1699-1706, 1999.

[6] A. G. Whittington, A. M. Hofmeister, and P. I. Nabelek. Temperature-dependent thermal diffusivity of the Earth's crust and implications for magmatism. Nature, 458:319-321, 2009.

[7] C. Forsberg. The advanced high-temperature reactor: high-temperature fuel, liquid salt coolant, liquid-metal-reactor plant. Progress in Nuclear Energy, 47(1-4):32-43, 2005.

[8] C. Le Brun. Molten salts and nuclear energy production. J. Nucl. Mater., 360:1-5, 2007.

[9] S. Jahn and P.A. Madden. Modeling Earth materials from crustal to lower mantle conditions: a transferable set of interaction potentials for the CMAS system. Phys. Earth Planet. Inter., 162:129-139, 2007.

[10] N. de Koker. Thermal conductivity of $\mathrm{MgO}$ periclase from equilibrium first principles molecular dynamics. Phys. Rev. Lett., 103:125902, 2009.

[11] S. Stackhouse, L. Stixrude, and B. B. Karki. Thermal conductivity of periclase (MgO) from first principles. Phys. Rev. Lett., 104:208501, 2010.

[12] S. P. de Groot and P. Mazur. Non-equilibrium Thermodynamics. Dover, New York, 1984.

[13] N. Galamba, C. A. Nieto de Castro, and J. F. Ely. Equilibrium and nonequilibrium molecular dynamics simulations of the thermal conductivity of molten alkali halides. J. Chem. Phys., 126(20):204511, 2007.

[14] P. Sindzingre and M. J. Gillan. A computer-simulation study of transport coefficients in alkalihalies. J. Phys.: Condens. Matter, 2(33):7033-7042, 1990.

[15] R.J. Heaton, R. Brookes, P.A. Madden, M. Salanne, C. Simon, and P. Turq. A first-principles description of liquid $\mathrm{BeF}_{2}$ and its mixtures with $\mathrm{LiF}: 1$. potential development and pure $\mathrm{BeF}_{2}$. J. Phys. Chem. B, 110(23):11454-11460, 2006.

[16] M. Salanne, C. Simon, P. Turq, and P.A. Madden. Conductivity-viscosity-structure: unpicking the relationship in an ionic liquid. J. Phys. Chem. B, 111(18):4678-4684, 2007.

[17] M. Salanne, C. Simon, P. Turq, and P.A. Madden. Heat-transport properties of molten fluorides: determination from first-principles. J. Fluorine Chem., 130(1):38-44, 2009.

[18] M. Salanne, C. Simon, H. Groult, F. Lantelme, T. Goto, and A. Barhoun. Transport in molten LiF-NaF-Z $\mathrm{rF}_{4}$ mixtures: a combined computational and experimental approach. J. Fluorine Chem., 130(1):61-66, 2009.

[19] B. Rotenberg, M. Salanne, C. Simon, and R. Vuilleumier. From localized orbitals to material properties: building classical force fields for nonmetallic condensed matter systems. Phys. Rev. Lett., 104(13):138301, 2010.

[20] A. V. Petrov, N. S. Tsypkina, and V. E. Seleznev. The behaviour of lattice thermal conductivity of crystals at high temperatures. High. Temp.-High. Pres., 8:537-542, 1976.

[21] A. Perronace, G Ciccotti, F. Leroy, A. H. Fuchs, and B. Rousseau. Soret coefficient for liquid argon-krypton mixtures via equilibrium and nonequilibrium molecular dynamics: A comparison with experiments. Phys. Rev. E, 66:031201, 2002.

[22] W. R. MacPherson and H. H. Schloessin. Lattice and radiative thermal conductivity variations through high P, T polymorphic structure transitions and melting points. Phys. Earth Planet. Inter., 29:58-68, 1982.

[23] R. G. Ross, P. Andersson, B. Sundqvist, and G. Bäckström. Thermal conductivity of solids and liquids under pressure. Rep. Prog. Phys., 47(1347-1402), 1984.

[24] P. J. D. Lindan and M. J. Gillan. A molecular dynamics study of the thermal conductivity of $\mathrm{CaF}_{2}$ and $\mathrm{UO}_{2}$. J. Phys.: Condens. Matter, 3:3929-3939, 1991. 
[25] O. Adjaoud, G. Steinle-Neumann, and S. Jahn. $\mathrm{Mg}_{2} \mathrm{SiO}_{4}$ liquid under high pressure from molecular dynamics. Chem. Geol., 256(3-4):185-192, 2008.

[26] M. Wilson, P. A. Madden, M. Hemmati, and C. A. Angell. Polarization effects, network dynamics, and the infrared spectrum of amorphous $\mathrm{SiO}_{2}$. Phys. Rev. Lett., 77(19):4023-4026, 1996. 